\title{
Genetic variants of microsomal epoxide hydrolase and glutamate-cysteine ligase
} in COPD

\author{
S. Chappell, L. Daly, K. Morgan, T. Guetta-Baranes, J. Roca, R. Rabinovich, J. Lotya, \\ A.B. Millar, S.C. Donnelly, V. Keatings, W. MacNee, J. Stolk, P.S. Hiemstra, \\ M. Miniati, S. Monti, C.M. O'Connor and N. Kalsheker
}

ABSTRACT: The genetic factors that contribute to the development of chronic obstructive pulmonary disease (COPD) are poorly understood. Many candidate genes have been proposed, including enzymes that protect the lung against oxidative stress, such as microsomal epoxide hydrolase (EPHX1) and glutamate-cysteine ligase (GCL). To date, most reported findings have been for EPHX1, particularly in relation to functional variants associated with fast and slow metabolism of epoxide intermediates. The present study aimed to identify any association of variation in these genes with COPD susceptibility or severity.

In total, 1,017 white COPD patients and 912 nondiseased age and sex matched smoking controls were genotyped for six single nucleotide polymorphisms (SNPs) in EPHX1 (including the fast and slow variants and associated haplotypes), and eight SNPs in the two genes encoding GCL. GCL is a rate-limiting enzyme in the synthesis of glutathione, a major contributor to antioxidant protection in the lung.

No association of variation was found in EPHX1 or GCL with susceptibility to COPD or disease severity.

This is the largest reported study to date and is well powered to detect associations that have been previously suggested. The current data indicate that these genetic variants are unlikely to be related to susceptibility or disease severity in white chronic obstructive pulmonary disease patients.

KEYWORDS: Chronic obstructive pulmonary disease, genetic susceptibility, glutamate-cysteine ligase, microsomal epoxide hydrolase

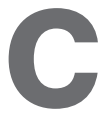

hronic obstructive pulmonary disease (COPD) is the sixth leading cause of morbidity and mortality in the western world and the prevalence of the disease is increasing as the population ages [1]. Cigarette smoking is the major environmental contributor to the disease in western societies yet only $15 \%$ of cigarette smokers develop the disease, indicating that genetic factors play a part in determining susceptibility. With the notable exception of severe $\alpha_{1}$-antitrypsin $\left(\alpha_{1}\right.$-AT) deficiency, these genetic factors are poorly understood.

Given that $>95 \%$ of those who develop COPD are smokers and oxidative stress is thought to be important in the pathogenesis of the disease, genetic variation in enzymes that protect the lung against smoke-induced oxidative stress has been a significant focus of study. Microsomal epoxide hydrolase (EPHX1), an enzyme involved in the first-pass metabolism of epoxide intermediates, has received particular attention as two functional variants of the gene, which confer slow and fast metabolic activity, have been identified [2] The slow variant decreases activity by $\sim 40-50 \%$, whilst the fast variant increases activity by $\sim 25 \%$. Since the initial report of an association between increased susceptibility to COPD and His-His homozygosity at the "slow" variant locus (Tyr113His in exon 3) [3], subsequent studies have variously supported this association [4-6], or have reported an association with disease severity rather than susceptibility [7]; observed no association between this polymorphism and

This manuscript has supplementary data accessible from www.ers.ersjournals.com

AFFILIATIONS

For affiliations, see

Acknowledgements section.

CORRESPONDENCE

N. Kalsheker

Division of Clinical Chemistry

University Hospital

Nottingham

NG7 2UH

UK

Fax: 441158230722

E-mail:noor.kalsheker@

nottingham.ac.uk

Received

April 292008

Accepted after revision:

June 192008

SUPPORT STATEMENT

The present work was supported by the European Union 5th Framework

Programme under contract QLG1-CT-

2001-01012 (COPD GENE the SCAN

project) and is protected as

intellectual property. S.C. Donnelly

was supported by Science

Foundation Ireland (Dublin, Ireland).

J. Lotya was supported by a grant

from the Health Research Board

(Dublin).

STATEMENT OF INTEREST

None declared. 
disease susceptibility or severity [8-10]; or reported a protective effect of this polymorphism [11]. Similar disparities in study outcomes are reported for the "fast" variant (His139Arg in exon 4) with possession of the minor variant reported as being associated with protection $[12,13]$ or having no effect $[3,11]$.

The lack of replication and inconsistency in these studies most likely reflect a range of issues that can produce false-positive or false-negative outcomes in case-control studies of complex diseases where relatively weak associations between single genes and disease are expected [14]. These include lack of power due to insufficient numbers of cases and controls, poor matching of cases and controls, particularly for smoking [15], and genetic heterogeneity in and between different study populations. A recent study has also shown that, when using restriction fragment length polymorphism (RFLP) techniques, genotyping error arising from the presence of a single nucleotide polymorphism (SNP) at Lys119 near the exon 3 slow polymorphism can lead to significant overestimation of His113 homozygotes [10] with resulting lack of HardyWeinberg (HW) equilibrium in study populations. Indeed, in a meta-analysis of previous studies in which populations that were not in HW equilibrium were omitted, His113 homozygosity was reported as being protective against the development of COPD [11].

To address many of these issues, in a previous study the present authors collected samples from a cohort of 1,017 white COPD patients and 912 nondiseased control smokers, matched for ethnicity, age and sex [16]. This represented the largest collection reported to date in a candidate gene approach. By matching cases and controls from each centre in Europe the risk of population stratification was minimised. Recent data from genome-wide association studies have shown that, at least in the British population, there is very little evidence for stratification in nearly 3,000 controls analysed to date. A relatively small number of genes showed significant differences in allele frequencies, suggesting that the population is relatively homogeneous and not as variable as previously thought [17]. In the current study this resource was used to evaluate the relationship between EPHX1 variants with respect to both the occurrence and severity of COPD. The association between genetic variation in glutamate-cysteine ligase (GCL; also known as $\gamma$-glutamyl-cysteine synthetase) and risk of developing COPD was assessed. This is the rate-limiting enzyme in the synthesis of glutathione, a central contributor to the lung's anti-oxidant defence system. Recently, variation in the catalytic subunit of the gene has been shown to be associated with lung disease severity in cystic fibrosis [18]. To date, genetic variation in this gene has only been reported in one small study of COPD in which no association was found [19].

\section{MATERIALS AND METHODS \\ Subjects}

COPD cases and control subjects were recruited at six European centres. The number of subjects recruited from each centre was as follows. Barcelona (Spain): 70 controls and 138 cases; Bristol (UK): 152 controls and 129 cases; Dublin (Ireland): 195 controls and 196 cases; Edinburgh (UK): 81 controls and 168 cases; Leiden (the Netherlands): 216 controls and 188 cases; and Pisa (Italy): 198 controls and 198 cases.
Approval for the study was obtained from the appropriate committees at each recruitment centre. Informed consent was obtained from all subjects. Criteria for patient recruitment were: a firm clinical diagnosis of stable COPD; airflow limitation as indicated by forced expiratory volume in one second $(\mathrm{FEV} 1) \leqslant 70 \%$ normal predicted values; FEV1/forced vital capacity (FVC) $<70 \%$ pred; no significant reversibility on bronchodilation; and a smoking history of $\geqslant 20$ pack-yrs. Patients were excluded from the study if they had an established diagnosis of asthma, lung cancer, a history of atopy, known $\alpha_{1}$-AT deficiency or a serum $\alpha_{1}$-AT level $<1.0 \mathrm{~g} \cdot \mathrm{L}^{-1}$. Subjects were also excluded if they had had an acute exacerbation in the 4 weeks preceding assessment for the study.

Control subjects were recruited at each centre to match COPD patients for ethnicity, age, sex and smoking history. Exclusion criteria were as described for cases and also included a family history of COPD. Only individuals with no evidence of airflow obstruction (FEV1 and FVC $\geqslant 80 \%$ and $\mathrm{FEV} 1 / \mathrm{FVC}>70 \%$ ) were included in the control group. Only white subjects were recruited for cases and controls. Complete matching between cases and controls was not achieved, but this was taken into account during the analysis.

No previous evidence has been found for population stratification in this sample set, in keeping with the data obtained from a large sample of the British population [16, 17].

\section{SNP mapping and haplotypes}

DNA sequencing to identify SNPs was carried out using a previously described approach [16]. Primers were designed for PCR so that the exons and flanking regions of the genes could be screened. These SNPs were then genotyped using Taqman assays (Applied Biosystems, Foster City, CA, USA) in 291 independent samples from white subjects from a previous study of COPD to obtain more robust data on the frequencies of SNPs. All subjects had given informed consent and the study was approved by the ethics committee of each recruiting centre. Where SNPs were in full linkage disequilibrium in the sequenced samples, a single representative SNP was chosen for genotyping. Where the minor allele frequency of SNP fell below $5 \%$ it was removed from further analyses.

\section{Genotyping of study samples}

Genotyping of the study population for six SNPs in the EPHX1 gene and eight SNPs in the GCL genes (five in the GCL catalytic (GCLC) and three in the GCL modifier (GCLM) subunits) was carried out at Geneservices Ltd (Cambridge, UK) using Taqman assays. Primer and probe sequences are available on request. As a quality control measure, the 44 samples of known genotype from sequencing were included. These genotypes were unknown to Geneservices Ltd but were used as an internal quality control measure. If discrepancies were noted the analysis was repeated; this resulted in $100 \%$ concordance for all the assays.

\section{Statistical analysis}

Analysis of genetic variation in population groups

Each of the SNPs in the three genes were analysed for HW equilibrium. To examine linkage disequilibrium, the correlation coefficient between SNP pairs within each gene in cases and 
controls was calculated. As matching was not completely achieved on recruitment, allele and genotype frequencies were adjusted for any residual confounding due to age, sex, smoking and centre using logistic regression. This also gave an increase in power by accounting for the matched design. Interactions of age, sex and smoking with centre were included as previously described [16]. Due to the study size it was possible to detect small differences between cases and controls. For instance, over the SNPs investigated, the (rare-type) allele frequency in controls ranged from a low of $7.4 \%$ to a high of $26.6 \%$ (both SNPs being in the GCL gene; tables 1 and 2). Using these extremes as examples and taking a $1 \%$ two-sided level of significance, the study had a power of $95 \%$ to detect an allele frequency of $\geqslant 11.4 \%$ in the cases compared with $7.4 \%$ in controls, and a frequency of $\geqslant 32.8 \%$ compared with $26.6 \%$. The corresponding detectable percentages for a lower frequency in cases than controls are 4.2 and $20.8 \%$, respectively.

\section{Haplotype analysis}

For full analysis of the relationships between COPD and EPHX1 and GCL variants, a staged, haplotype-based approach was used, as described previously [16]. First, the SNP groupings on which to perform the haplotype analysis were identified. To allow for any combination, the present authors screened all the possible SNP groups in the EPHX1 gene and in the GCLM and GCLC genes for differences between cases and controls. This was carried out by performing an omnibus test [20] and employing a p-value of $<0.01$ as a cut-off value for statistical significance. Exact p-values based on 10,000 permutations were generated. Extremely rare haplotypes, occurring at a frequency of $<1 \%$ within each of these groups, were excluded. Individual haplotypes were compared between COPD cases and controls using the pseudo Chi-squared test. A $1 \%$ level of significance was taken.

\begin{tabular}{|c|c|c|c|c|c|c|}
\hline TABLE & $\begin{array}{l}\text { Gluta } \\
\text { frequ } \\
\text { pulm }\end{array}$ & $\begin{array}{l}\text { nate-cystei } \\
\text { encies in cc } \\
\text { nary disea }\end{array}$ & $\begin{array}{l}\text { ine ligas } \\
\text { ontrols a } \\
\text { se (COF }\end{array}$ & $\begin{array}{l}\text { e (GC } \\
\text { ind ch } \\
\text { D) su }\end{array}$ & $\begin{array}{l}\text { allele } \\
\text { nic ob } \\
\text { ects }\end{array}$ & structive \\
\hline \multirow[t]{2}{*}{ SNP no. } & \multirow[t]{2}{*}{ SNP } & \multirow{2}{*}{$\begin{array}{l}\text { dbSNP } \\
\text { reference }\end{array}$} & \multirow[t]{2}{*}{ Control } & \multirow[t]{2}{*}{ COPD } & \multicolumn{2}{|c|}{$\mathrm{p}$-value } \\
\hline & & & & & Crude & Corrected ${ }^{\#}$ \\
\hline \multicolumn{7}{|l|}{ GCLC } \\
\hline $1 c$ & C-129T & rs 17883901 & 0.074 & 0.068 & 0.48 & 0.67 \\
\hline $2 c$ & G24434A & rs2100375 & 0.279 & 0.277 & 0.86 & 0.65 \\
\hline $3 c$ & G36138A & rs16883912 & 0.102 & 0.102 & 0.95 & 0.41 \\
\hline $4 c$ & A37764G & rs1555903 & 0.091 & 0.087 & 0.62 & 0.58 \\
\hline $5 c$ & G39514A & rs524553 & 0.222 & 0.231 & 0.50 & 0.15 \\
\hline \multicolumn{7}{|l|}{ GCLM } \\
\hline $1 \mathrm{~m}$ & C-590T & rs41303970 & 0.159 & 0.152 & 0.62 & 0.30 \\
\hline $2 m$ & С7623Т & rs7515191 & 0.354 & 0.336 & 0.26 & 0.21 \\
\hline $3 m$ & G14613T & rs769211 & 0.266 & 0.250 & 0.29 & 0.25 \\
\hline
\end{tabular}

\section{RESULTS}

\section{Characteristics of the study population}

The characteristics of the control and COPD groups recruited to the study are summarised in table 3. Targeted recruitment of controls yielded groups with similar age and sex profiles, although complete matching of control and COPD groups was not achieved as a high proportion of smokers aged $>65 \mathrm{yrs}$ had evidence of some obstruction in pulmonary function and were therefore excluded from recruitment as controls. This incomplete matching was adjusted for in the analysis of the data. Within the COPD group, those recruited were almost equally distributed across the Global Obstructive Lung Disease severity categories of moderate, severe and very severe (see table $1 S$ of online supplementary data).

\section{SNP mapping}

EPHX1

In total, 25 SNPs were identified in the EPHX1 gene on sequencing (see table $2 \mathrm{~S}$ of online supplementary data). Two of these SNPs (rs45616640 and rs45505095) had a minor allele frequency of $<5 \%$ so were excluded from further analysis. Two further SNPs (rs45467394 and rs4149227) were unsuitable for Taqman assay design. Complete linkage disequilibrium was observed between SNPs at sites -699/-613/-362 and -290/-200 in the $5^{\prime}$ flanking region leaving 19 SNPs with poor linkage disequilibrium occurring at a frequency of $>5 \%$. As screening for 19 SNPs would result in a large number of haplotypes occurring at a frequency $<1 \%$, thus reducing the power of the study, a selection of six SNPs was made based on either previous associations or with potential functional variants. The SNPs chosen included three that fully covered variation in the promoter region (rs2854450, rs2854451 and rs3753658), the slow and fast functional SNPs (rs1051740 and rs2234922, respectively) that have been shown to affect activity of EPHX1 and one SNP (rs4653695) from the 3' untranslated end of the gene.

\section{GCL}

Six SNPs were identified in the GCLC subunit and six in the GCLM subunit. The location and nucleotide substitution of these SNPs are indicated in the supplementary data (table 3S). In the GCLC subunit, two SNPs (rs2100375 and rs1901773) were in complete linkage disequilibrium, so only the rs2100375 was assessed in further analyses. In 291 independent samples, the remaining five SNPs were found to contribute to nine haplotypes that occurred with a frequency of $>1 \%$ and accounted for $99 \%$ of the observed variation in the gene, thus, providing almost complete coverage of the gene. These five SNPs were genotyped in the study population. In the GCLM subunit, three SNPs (rs41303970, rs743119 and rs2273406) were in complete linkage disequilibrium, so rs41303970 was chosen to infer information for all three sites in subsequent analyses. SNP rs35267053 had a minor allele frequency of $<5 \%$, so was not included in further investigations. The three remaining SNPs were found to contribute to four haplotypes that occurred with a frequency of $>1 \%$ and accounted for $99 \%$ of the observed variation in the 291 samples screened. All three SNPs were genotyped in the final sample resource.

\section{Analysis of variation in EPHX1 and GCL genes in COPD and controls}

The allele and genotype frequencies of the six EPHX1 SNPs that were screened in the full study population are shown in 


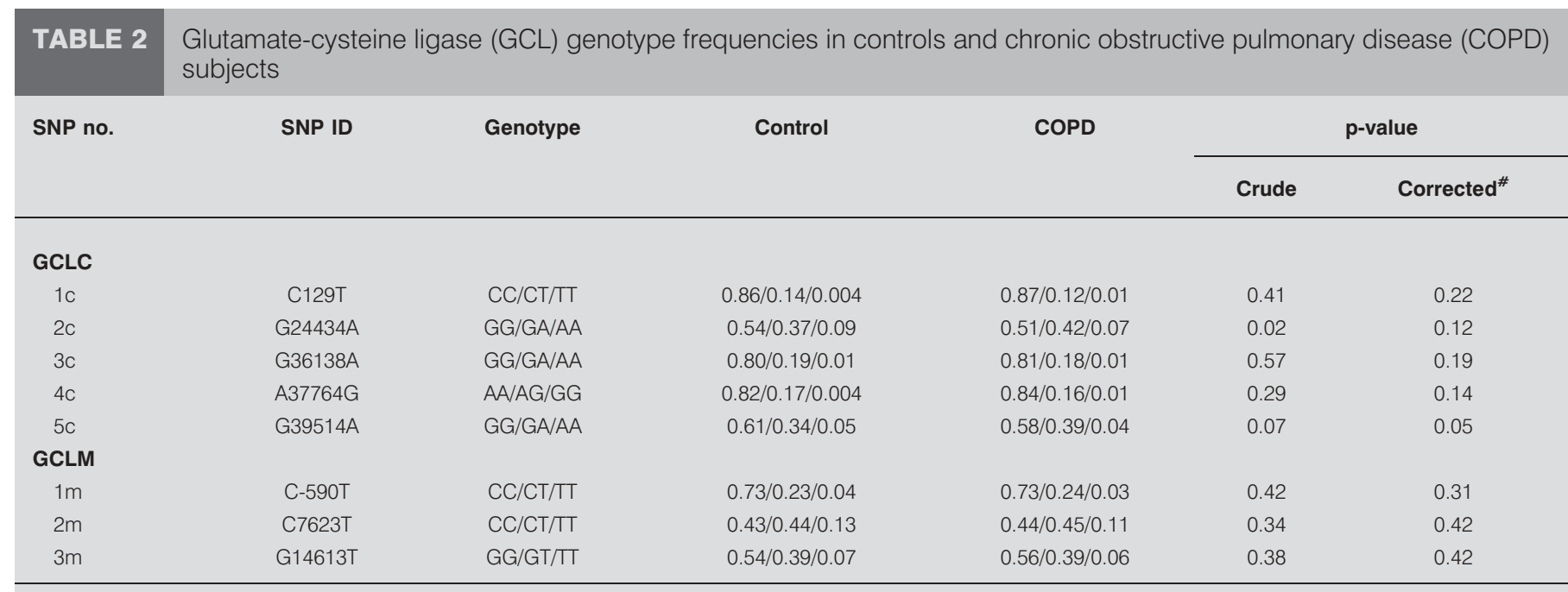

SNP: single nucleotide polymorphism; GCLC: GCL catalytic subunit; GCLM: GCL modifier subunit. " : adjusted by logistic regression for age, sex, centre and smoking

tables 4-6. For ease of presentation, screened SNPs are numbered sequentially from 1 to 6 with the gene location relative to the transcription start site, base substitution and the National Centre for Biotechnology Information SNP database (dbSNP) reference number for each SNP indicated. All of the SNPs were within the accepted criteria for HW equilibrium and the frequencies of the SNPs were similar in the populations from all six recruitment centres.

No difference in allele frequency was observed between patient and control groups for any individual EPHX1 SNP nor was there any difference (at the $1 \%$ level set for acceptance of significance) in SNP genotype frequencies between patients and controls (table 4-6). Similarly, when classified into functional phenotypes on the basis of genotype at the slow and fast loci, as described by SMITH and HARRISON [3], no difference was observed between controls and COPD patients. Neither were functional phenotypes found to be associated with disease severity within the cases (see table $4 \mathrm{~S}$ of online supplementary data). The common six SNP haplotypes (occurring with a frequency $>1 \%$ ) associated with the slow and fast functional variants were also evaluated, but no difference was found in haplotypes between control and COPD groups (table 7).

As with the EPHX1 gene, the frequencies of the six SNPs screened in the GCLC and GCLM genes were similar in the populations from all six recruitment centres and, with the exception of SNP 1m (C-590T GLCM subunit), all were within the accepted criteria for HW equilibrium. For SNP 1m, the frequency of the minor allele homozygotes was slightly higher than expected (4.3 versus $2.5 \%$ ) in the control population $(p=0.002)$. This was unlikely to be due to genotyping error as the blinded controls for this assay were accurately assigned. The frequency of the minor allele is $<5 \%$ and small deviations in observed numbers may cause significant deviation from HW equilibrium. The possibility of a copy number variant, which often contributes to deviations from HW equilibrium, cannot be fully excluded although no evidence for this was found in preliminary experiments using real-time PCR (data not shown).
No difference in allele frequency was observed between case and control groups for any individual SNP in either the GCLC or GCLM genes, nor was there any difference in SNP genotype frequencies (tables 1 and 2).

Linkage disequilibrium analysis (see tables 5S and 6S in the online supplementary data) demonstrated strong linkage between SNPs 2c and 5c (rs2100375 and rs524553) in the GCLC subunit and between all three SNPs in the GCLM subunit in both case and control groups. No strong linkage was observed between SNPs evaluated in the EPHX1 gene in either cases or controls.

Haplotype analysis of SNP combinations in each of the three genes did not identify any combinations that were related to COPD. Similarly, when evaluated on the basis of disease severity within the COPD group, no association between haplotypes and disease severity was observed for any of the three genes evaluated.

\section{DISCUSSION}

The EPHX1 gene has been a significant focus for studies on genetic susceptibility in COPD in the past $10 \mathrm{yrs}$. It is a promising candidate gene as it is strongly expressed in the

\begin{tabular}{lccc} 
TABLE 3 & \multicolumn{4}{c}{$\begin{array}{l}\text { Characteristics of control and chronic obstructive } \\
\text { pulmonary disease (COPD) study subjects }\end{array}$} \\
& Control & COPD & p-value \\
\hline Subjects $\mathbf{n}$ & 912 & 1017 & \\
Male \% & 63.6 & 69.8 & 0.0038 \\
Age yrs & $60.8 \pm 8.9$ & $65.9 \pm 8.2$ & $<0.0001$ \\
Smoking pack-yrs & $38.7 \pm 17.4$ & $48.7 \pm 22.8$ & $<0.0001$ \\
FEV 1 \% pred & $95.4 \pm 11.0$ & $43.2 \pm 15.2$ & $<0.0001$ \\
FEV 1 /FVC & $77.8 \pm 4.9$ & $47.6 \pm 12.1$ & $<0.0001$ \\
\hline
\end{tabular}

Data are presented as mean $\pm S D$, unless otherwise stated. FEV 1 : forced expiratory volume in one second; \% pred: \% predicted; FVC: forced vital capacity. 


\begin{tabular}{|c|c|c|c|c|c|c|c|}
\hline \multicolumn{2}{|c|}{ TABLE 4} & \multicolumn{6}{|c|}{$\begin{array}{l}\text { Microsomal epoxoide hydrolase minor allele } \\
\text { frequencies in controls and chronic obstructive } \\
\text { pulmonary disease (COPD) subjects }\end{array}$} \\
\hline \multirow{2}{*}{$\begin{array}{l}\text { SNP } \\
\text { no. }\end{array}$} & \multirow[t]{2}{*}{ SNP I } & \multirow{2}{*}{\multicolumn{2}{|c|}{$\begin{array}{l}\text { dbSNP } \\
\text { reference }\end{array}$}} & \multirow[t]{2}{*}{ Control } & \multirow[t]{2}{*}{ COPD } & \multicolumn{2}{|c|}{$p$-value } \\
\hline & & & & & & Crude & Corrected $^{\#}$ \\
\hline 1 & $C-3 S$ & & rs2854450 & 0.217 & 0.206 & 0.40 & 0.30 \\
\hline 2 & G-36 & & rs2854451 & 0.245 & 0.242 & 0.82 & 0.95 \\
\hline 3 & G-2 & & rs3753658 & 0.124 & 0.132 & 0.43 & 0.63 \\
\hline 4 & T66 & & rs1051740 & 0.301 & 0.294 & 0.60 & 0.86 \\
\hline 5 & A134 & $26 G$ & rs2234922 & 0.204 & 0.208 & 0.76 & 0.90 \\
\hline 6 & A200 & $94 \mathrm{C}$ & rs4653695 & 0.107 & 0.111 & 0.65 & 0.91 \\
\hline
\end{tabular}

SNP: single nucleotide polymorphism; dbSNP: SNP database. ${ }^{\#}$ : adjusted by logistic regression for age, sex, centre and smoking.

bronchial epithelium and its biological role in detoxifying epoxides is extremely relevant in protecting the lung against smoke-induced damage. In addition, there is significant individual variation in enzymatic activity, and genetic polymorphisms that modify enzymatic activity have been identified [21]

The majority of studies on genetic variants of EPHX1 in COPD have evaluated polymorphisms in the coding region of the gene that are associated with slow (Tyr113His) and fast (His139Arg) enzymatic activity, with conflicting results. Many of these studies have limitations with respect to power (with numbers of subjects in the case and control groups ranging 60-200); matching for ethnicity, age, sex and smoking history also vary considerably. In addition, studies that analysed polymorphic variation using RFLP techniques are likely to have overestimated the proportion of slow (113His) homozygotes [10].

The present study overcomes many of these limitations by evaluating EPHX1 polymorphisms in case and control groups with sufficient power to readily replicate previously reported associations. All subjects were white and any potential effects of population stratification were minimised by recruiting cases and controls from the same populations. In addition, targeted recruitment of controls facilitated the enrolment of controls with a similar profile to COPD patients with respect to sex, age and smoking.
Analysis of EPHX1 polymorphisms showed no relationship between susceptibility to COPD and variation at the Tyr113His or His139Arg loci. Given the significantly greater level of power and case-control matching in the present study it is unlikely that effects of the magnitude observed in previous, less powered studies have been missed. All EPHX1 SNPs evaluated were in HW equilibrium in control populations. This, along with the inclusion of previously sequenced samples as quality controls in the genotyping analysis, indicates that the current analysis is not affected by genotyping error.

Furthermore, detailed analysis of haplotypes corresponding to the fast (His139Arg) and slow (Tyr113His) variants failed to identify haplotypes that were associated with disease. The present authors also analysed variation in the $5^{\prime}$ promoter region of the EPHX1 gene, which has been shown to modulate enzyme expression by up to $30 \%$ [21], but no association with disease susceptibility was found.

The current results are at variance with two adequately powered recent studies which report the protective effects of the His139Arg [12] and Try113His [11] alleles, although neither study replicated the other's results. In a study of 304 emphysema patients and 441 controls, HeRSH et al. [12] report a protective effect (odds ratio 0.73, $p=0.03$ ) with the fast variant of codon 139, while no association was observed with variation in the 113 codon. By contrast, BROGGER et al. [11] evaluated the same polymorphisms in 492 white smokers and ex-smokers (244 COPD patients and 248 controls) and reported a protective effect of His/His homozygosity at the 113 locus, with no effect of variation at the 139 locus. The present study did not replicate either of these observations, nor were they noted in the majority of the smaller studies [3-10].

Sequencing of the EPHX1 gene revealed that variation in this gene is high, with 25 polymorphisms being identified. This may explain some of the conflicting results reported in studies of the slow and fast variants as these polymorphisms could contribute to disease susceptibility on a background of other specific SNP variants or haplotypes. However, this seems unlikely as the present authors have captured the most significant haplotypes corresponding to the fast and slow variants. It may be that variation in other genes plays a role as studies on the impact of the slow and fast EPHX1 genetic variants indicate that they may have only a modest impact on in vivo activity levels and that

TABLE 5 Microsomal epoxoide hydrolase genotype frequencies in controls and chronic obstructive pulmonary disease (COPD) subjects

\begin{tabular}{|c|c|c|c|c|c|c|}
\hline \multirow[t]{2}{*}{ SNP no. } & \multirow[t]{2}{*}{ SNP ID } & \multirow[t]{2}{*}{ Genotype } & \multicolumn{2}{|c|}{ Genotype frequency } & \multicolumn{2}{|c|}{$\mathrm{p}$-value } \\
\hline & & & Control & COPD & Crude & Corrected $^{\#}$ \\
\hline 1 & C-399T & $\mathrm{CC} / \mathrm{CT} / \mathrm{TT}$ & $0.62 / 0.33 / 0.05$ & $0.63 / 0.33 / 0.04$ & 0.50 & 0.17 \\
\hline 2 & G-362A & GG/GA/AA & $0.57 / 0.36 / 0.06$ & $0.58 / 0.35 / 0.07$ & 0.83 & 0.98 \\
\hline 3 & G-290T & GG/GT/TT & $0.77 / 0.21 / 0.02$ & $0.75 / 0.23 / 0.02$ & 0.27 & 0.12 \\
\hline 4 & T6651C & $\pi / T C / C C$ & $0.49 / 0.41 / 0.10$ & $0.50 / 0.41 / 0.09$ & 0.80 & 0.98 \\
\hline 5 & A13426G & $\mathrm{AA} / \mathrm{AG} / \mathrm{GG}$ & $0.64 / 0.31 / 0.05$ & $0.63 / 0.33 / 0.04$ & 0.72 & 0.97 \\
\hline 6 & A20094C & $\mathrm{AA} / \mathrm{AC} / \mathrm{CC}$ & 0.80/0.19/0.01 & $0.79 / 0.21 / 0.01$ & 0.30 & 0.43 \\
\hline
\end{tabular}

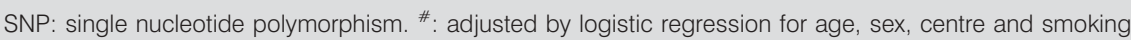




\begin{tabular}{|c|c|c|c|c|}
\hline \multirow{2}{*}{$\begin{array}{l}\text { TABLE } 6 \\
\text { Phenotype }\end{array}$} & \multicolumn{4}{|c|}{$\begin{array}{l}\text { Microsomal epoxoide hydrolase functional } \\
\text { phenotype frequencies in controls and chronic } \\
\text { obstructive pulmonary disease (COPD) subjects }\end{array}$} \\
\hline & SNP4/SNP5 genotype & Control & COPD & p-value \\
\hline Normal & TT/AA or $T C / A G$ & 0.433 & 0.453 & 0.37 \\
\hline Slow & TC/AA or CC/AA & 0.364 & 0.345 & 0.41 \\
\hline Fast & $T T / A G$ or $T T / G G$ & 0.204 & 0.201 & 0.90 \\
\hline \multicolumn{5}{|c|}{$\begin{array}{l}\text { SNP: single nucleotide polymorphism; normal: no mutation in gene (exon 3:Tyr/ } \\
\text { Tyr and exon 4: His/His), or heterozygote for both exon } 3 \text { (Tyr113/His113) and } \\
\text { exon } 4 \text { (His139/Arg139) mutations; slow: one slow allele (exon 3: Tyr113/ } \\
\text { His113) or two slow alleles; fast: at least one fast mutation (exon 4: His139/ } \\
\text { Arg139 or Arg139/Arg139) and no exon } 3 \text { mutation (Tyr113/Tyr113). }\end{array}$} \\
\hline
\end{tabular}

post-transcriptional modification may be important [21-23]. Therefore, it seems unlikely that these particular variants contribute to COPD susceptibility, although the possibility of rare variants playing a role cannot be excluded and significantly larger studies are needed to test this reliably.

The rate limiting enzyme in glutathione synthesis, GCL, is also a potential candidate gene for susceptibility to COPD. Significantly decreased glutathione levels are observed in the lungs of GCLM knockout mice [24] and message expression for this subunit is decreased in alveolar macrophages from healthy smokers [25]. In addition, the presence of GCL protein in the bronchial airways appears to be diminished in smokers and COPD patients compared with nonsmoking controls [26, 27]. Both subunits of this enzyme were genotyped and investigated with the role of the genetic variation observed as a potential risk factor for disease. As with EPHX1, no association was found with disease and SNPs in genes encoding either subunit of the GCL enzyme. This confirms the only other reported study of GCL genetic variation in COPD, which investigated a single SNP in the GCLC gene (rs17883901; SNP 1c) in 322 subjects from the Han Chinese population, and failed to find evidence for an association with COPD [19]. The present study has extended these previous findings to include more SNPs throughout the GCLC gene, as well as considering variation in the gene for the GCLM subunit.

In conclusion, no evidence was found for a significant association of polymorphisms in microsomal epoxide hydrolase or glutamate-cysteine ligase with susceptibility to chronic obstructive pulmonary disease in a white population.

\section{ACKNOWLEDGEMENTS}

The authors would like to thank the following. For assisting with recruitment: A. Hann (University of Bristol, Bristol, UK); B. Callaghan and G. Hogan (University Colleage Dublin, Dublin, Ireland); J. Barr (University of Edinburgh, Edinburgh, UK); and C. Kolster-Bijdevaate (Leiden University Medical School, Leiden, the Netherlands). For clinical and technical assistance: G. Catapano, E. Fornai and C. Carli (University of Pisa, Pisa, Italy). For assistance in data analysis: E. Daly (University College Dublin). For comments on data analysis: B. Veldhuisen and J.J. Houwing (Leiden University Medical School).

\begin{tabular}{|c|c|c|c|c|}
\hline TABLE 7 & & $\begin{array}{l}\text { es assoc } \\
\text { e slow } 66 \\
\text { variants }\end{array}$ & $\begin{array}{l}\text { with m } \\
\text { (113His }\end{array}$ & $\begin{array}{l}\text { hal epoxide } \\
\text { ast } 13426 \mathrm{G}\end{array}$ \\
\hline \multirow[t]{2}{*}{ Haplotype } & & \multicolumn{2}{|c|}{ Frequency } & \multirow[t]{2}{*}{ p-value } \\
\hline & & Controls & COPD & \\
\hline \multicolumn{5}{|c|}{$6651 \mathrm{C}$ (113His) } \\
\hline CAGCAA & & 0.0998 & 0.0955 & 0.613 \\
\hline CGGC̄AA & & 0.0818 & 0.0775 & 0.5974 \\
\hline TGḠ̄AA & & 0.0262 & 0.0349 & 0.1197 \\
\hline CGTC̄AA & & 0.0195 & 0.0142 & 0.2036 \\
\hline \multicolumn{5}{|c|}{$13426 \bar{G}$ (139Arg) } \\
\hline CGGTGA & & 0.0656 & 0.0725 & 0.3944 \\
\hline TGGT馬 & & 0.0488 & 0.0357 & 0.0425 \\
\hline 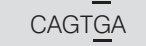 & & 0.0247 & 0.0285 & 0.3959 \\
\hline
\end{tabular}

COPD: chronic obstructive pulmonary disease. Relevant nucleotide substitutions are underlined. None of the haplotypes were significantly different between COPD and controls. Haplotypes with frequencies $<1 \%$ in both cases and controls are not shown.

Affiliations are as follows. S. Chappell, K. Morgan, T. GuettaBaranes and N. Kalsheker: Division of Clinical Chemistry, Molecular Medical Sciences, Institute of Genetics, University Hospital, University of Nottingham, Queens Medical Centre, Nottingham, UK; L. Daly and J. Lotya: School of Public Health and Population Science, University College Dublin, Ireland; J. Roca and R. Rabinovich: Pulmonary Services, Hospital Clinico y Provincial de Barcelona, Hospital Clinic, Barcelona, Spain; A.B. Millar: Lung Research Group, Dept of Clinical Science at North Bristol, University of Bristol, Southmead Hospital, Bristol, UK; S.C. Donnelly and C.M. O'Connor: School of Medicine and Medical Science, The Conway Institute, University College Dublin, Dublin, Ireland; V. Keatings: Letterkenny General Hospital, Letterkenny, County Donegal, Ireland; W. MacNee: Edinburgh Lung and the Environment Group Initiative Colt Laboratories, Medical Research Council Centre for Inflammation Research, Queen's Medical Research Institute, Edinburgh, UK; J. Stolk and P.S. Hiemstra: Dept of Pulmonology (C3-P), Leiden University Medical Center, Leiden, The Netherlands; M. Miniati and S. Monti: Italian National Research Council, Institute of Clinical Physiology, Pisa, Italy. Both C.M. O'Connor and N. Kalsheker contributed equally as lead authors.

\section{REFERENCES}

1 Pauwels RA, Rabe KF. Burden and clinical features of chronic obstructive pulmonary disease (COPD). Lancet 2004; 364: 613-620.

2 Hassett C, Aicher L, Sidhu JS, Omiecinski CJ. Human microsomal epoxide hydrolase: genetic polymorphism and functional expression in vitro of amino acid variants. Hum Mol Genet 1994; 3: 421-428.

3 Smith CA, Harrison DJ. Association between polymorphism in gene for microsomal epoxide hydrolase and susceptibility to emphysema. Lancet 1997; 350: 630-633. 
4 Park JY, Chen L, Wadhwa N, Tockman MS. Polymorphisms for microsomal epoxide hydrolase and genetic susceptibility to COPD. Int J Mol Med 2005; 15: 443-448.

5 Xiao D, Wang C, Du MJ, et al. Relationship between polymorphisms of genes encoding microsomal epoxide hydrolase and glutathione S-transferase P1 and chronic obstructive pulmonary disease. Chin Med J 2004; 117: 661-667.

6 Vibhuti A, Arif E, Deepak D, Singh B, Pasha MAQ. Genetic polymorphisms of GSTP1 and mEPHX correlate with oxidative stress markers and lung function in COPD. Biochem Biophys Res Comm 2007; 359: 136-142.

7 Yoshikawa M, Hiyama K, Ishioka S, Maeda H, Maeda A, Yamakido M. Microsomal epoxide hydrolase genotypes and chronic obstructive pulmonary disease in Japanese. Int J Mol Med 2000; 5: 49-53.

8 Takeyabu K, Yamaguchi E, Suzuki I, Nishimura M, Hizawa N, Kamakami Y. Gene polymorphism for microsomal epoxide hydrolase and susceptibility to emphysema in a Japanese population. Eur Respir J 2000; 15: 891-894.

9 Yim JJ, Park GY, Lee CT, et al. Genetic susceptibility to chronic obstructive pulmonary disease in Koreans: combined analysis of polymorphic genotypes for microsomal epoxide hydrolase and glutathione S-transferase M1 and T1. Thorax 2000; 55: 121-125.

10 Matheson CM, Raven J, Walters EH, Abramson MJ, Ellis JA. Microsomal epoxide hydrolase is not associated with COPD in a community-based sample. Hum Biol 2006; 78: 705-177.

11 Brogger J, Steen VM, Eiken HG, Gulsvik A, Bakke P. Genetic association between COPD and polymorphisms in TNF, ADRB2 and EPHX1. Eur Respir J 2006; 27: 682-688.

12 Hersh CP, Demeo DL, Lange C, et al. Attempted replication of reported chronic obstructive pulmonary disease candidate gene associations. Am J Respir Cell Mol Biol 2005; 33: 71-78.

13 DeMeo DL, Hersch CP, Hoffman EA, et al. Genetic determinants of emphysema distribution in the National Emphysema Treatment Trial. Am J Respir Crit Care Med 2007; 176: 42-48.

14 Molfino NA. Genetics of COPD. Chest 2004; 125: 1929-1940.

15 Lomas DA, Silverman EK. The genetics of chronic obstructive pulmonary disease. Respir Res 2001; 2: 20-26.
16 Chappell S, Daly L, Morgan K, et al. Cryptic haplotypes of SERPINA1 confer susceptibility to chronic obstructive pulmonary disease. Hum Mutat 2006; 2: 103-109.

17 Wellcome Trust Case Control Consortium. Genome-wide association study of 14,000 cases of seven common diseases and 3,000 shared. Nature 2007; 447: 661-678.

18 McKone EF, Shao J, Frangolias DD, et al. Variants in the glutamate-cysteine-ligase gene are associated with cystic fibrosis lung disease. Am J Respir Crit Care Med 2006; 174: 415-419.

19 Liu S, Zhou Y, Zong N, Ran P. Genetic analysis of CC16, OGG1, GCLC polymorphisms and susceptibility to COPD. Respirology 2007; 12: 29-33.

20 Zhao JH, Curris D, Sham PC. Model-free analysis and permutation tests for allelic associations. Hum Hered 2000; 50: 133-139.

21 Omiecinski CJ, Hassett C, Hosagrahara V. Epoxide hydrolase: polymorphism and role in toxicology. Toxicol Lett 2000; 15: 112-113.

22 Raaka S, Hassett C, Omiencinski CJ. Human microsomal epoxide hydrolase: 5'-flanking region genetic polymorphisms. Carcinogenesis 1998; 19: 387-393.

23 Hassett C, Lin J, Carty CL, Laurenzana EM, Omiecinski CJ. Human hepatic microsomal epoxide hydrolase: comparative analysis of polymorphic expression. Arch Biochem Biophys 1997; 337: 275-283.

24 Yang Y, Dieter MZ, Chen Y, Shertzer HG, Nebert DE, Dalton TP. Initial characterization of the glutamate-cysteine ligase modifier subunit Gclm(-/-) knockout mouse. Novel model system for a severely compromised oxidative stress response. J Biol Chem 2002; 277: 49446-49452.

25 Rahman I, MacNee W. Lung glutathione and oxidative stress: implications in cigarette smoke-induced airway disease. Am J Physiol 1999; 277: L1067-L1088.

26 Rahman I, van Schadewijk AA, Hiemstra PS, et al. Localization of $\gamma$-glutamylcysteine synthetase messenger RNA expression in lungs of smokers and patients with chronic obstructive pulmonary disease. Free Radic Biol Med 2000; 28: 920-925.

27 Harju T, Kaarteenaho-Wiik R, Soini Y, Sormunen R, Kinnula VL. Diminished immunoreactivity of $\gamma$-glutamylcysteine synthetase in the airways of smokers' lung. Am J Respir Crit Care Med 2002; 166: 754-759. 